\title{
Intelligent Based Terrain Preview Controller for a 3-axle Vehicle
}

\author{
J.T. Economou, D.J. Purdy, D. Galvão Wall, D. Diskett \& D. Simner \\ Centre for Defence Engineering, Cranfield University, Defence Academy of the United Kingdom, \\ Shrivenham, Swindon, SN6 8LA, UK.
}

\begin{abstract}
The paper presents a six-wheel half longitudinal model and the design of a dual level control architecture. The first (top) level is designed using a Sugeno fuzzy inference feedforward architecture with and without preview. The second level of controllers are locally managing each wheel for each axle. As the vehicle is moving forward the front wheels and suspension units will have less time to respond when compared to the middle and rear units, hence a preview sensor is used to compensate. The paper shows that the local active suspensions together with the Sugeno Fuzzy, (locally optimised using subtractive clustering), Feedforward control strategy is more effective and this architecture has resulted in reducing the sprung mass vertical acceleration and pitch accelerations.
\end{abstract}

\section{INTRODUCTION}

The design of conventional, passive, suspension systems is reaching a limit for high speed off-road conditions. One of the main users of fast off-road vehicles is the military, where it has shown (Sher et al. 1988) that one of the main contributors to survival is vehicle speed. A possible way of increasing the vehicle speed while reducing the vibration exposure of the crew is the use of an active suspension system combined with terrain preview. In a review of the literature (Sharp et al. 1995) it was noted that the use of terrain preview (Purdy et al. 2010),(Kiyotsugu 2003) together with an active suspension system would need to be used on "special test surfaces" for example; very rough (off-road) to be of any benefit. In this work a three axle military vehicle, Figure 1, is used to investigate the possible benefits of a Sugeno (Feng 2006),(Tagaki et al. 1985) based Fuzzy controller (using subtractive clustering) (Economou et al. 2006) to improve its performance at isolating the crew from the terrain. This work extends that done by (Mehrishi 2007),(Berrouila 2008),(Bo 2010),(Purdy et al. 2010) and (Purdy et al. 1997). Fuzzy Logic is a universal approximator, which enables modelling of complex process by input-output maps via a rule-base approach. In (Feng 2006) the historical ambiguity of fuzzy systems, although very successful in a wide range of industrial applications, is restored showing a state of the art survey of results offering not only guaranteed stability but also good performance. In (Economou et al. 2006), the use of optimization is explored in relation to fuzzy consequent variants and the derivation of the membership functions in relation to input-output data demonstrating that the fuzzy rules can be obtained in the presence of data. Hence the proposed fuzzy rule base (membership functions for example) are optimsed using fuzzy clustering methods as used in (Economou et al. 2006). The use of fuzzy logic in this paper is based on the Sugeno singleton fuzzy inference framework (Tagaki et al. 1985) providing bump-stop prediction based on look ahead terrain sensing Figure 1. This approach enables the use of a look ahead distance and thus time for a given vehicle velocity while acting at a pre-selected time depending on the suspension dynamics. This although in first instance appears similar to observer based solutions it differs because it prepares the vehicle to respond as well as possible for given known terrain profiles. The proposed control architecture results in the reduction of the sprung mass vertical accelerations (vertical, pitch) which consequently results in reducing the risk to injury to the vehicle passengers (Barnaby et al. 2009).

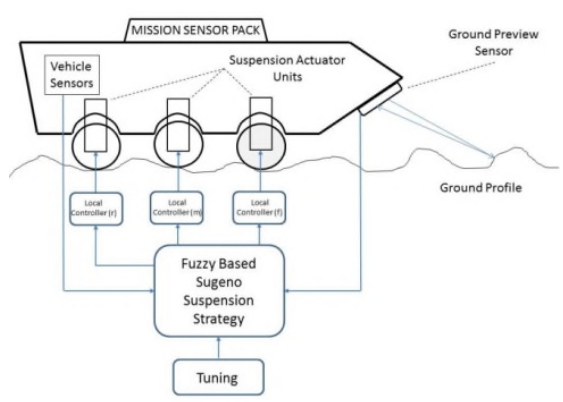

Figure 1. Overview of the Dual Level Control Architecture. 
It is shown in this paper that the use of fuzzy Sugeno (using subtractive clustering) feedfoward control architecture with preview data together with the active suspension system results in reducing the vertical and pitch sprung mass accelerations. Furthermore the analysis demonstrates the effect of the preview distance in relation to the controller performance.

\section{THEE AXLE VEHICLE MODELLING}

The active suspension for a single actuator system is shown in Figure 2, while the vehicle suspension for the tree axle vehicle system schematic is shown in Figure 3 respectively.

The vehicle model is based on the single wheel station model given in (Purdy et al. 1997) and used by (Mehrishi 2007, Berrouila 2008, Bo 2010, Purdy et al. 2010). The vehicle model is restricted to eight degrees of freedom, these being pitch and bounce of the vehicle body, vertical motion of each axle and one for each actuator, and is in effect a half vehicle model. The model is developed so that the simulation can be run with any combination of the suspension actuators being passive or active.

The suspension actuator is shown diagrammatically in Figure 2. In this diagram the motion of the top of the actuator is $z_{1}$ and the bottom $z_{2}$, the mechanical stiffness $k_{d}$, flow restriction through the damper valve $R$ and the floating and piston areas $a_{d}$ and $a_{p}$ respectively. The oil in the device is assumed to be incompressible.

Letting the motion across the actuator be;

$z_{w}=z_{1}-z_{2}$

The force generated by the actuator is;

$f_{a}=a_{p} P_{2}$

Where $P_{2}$ is the pressure above the piston.

The flow into the actuator;

$q=\dot{\eta} a_{d}+\left(P_{1}-P_{2}\right) / R$

Where $\dot{\eta}$ is the motion of the floating piston and $P_{1}$ is the pressure between the damper valve and the floating piston, which is given by;

$$
P_{1}=\eta k_{d} / a_{d}
$$

The flow through the damper value is given by;

$$
\dot{z}_{w} a_{p}=\left(P_{1}-P_{2}\right) / R
$$

These equations are manipulated to give the equations of motion for the actuator with the addition of bump and rebound stops to limit the suspension travel.
The validation of the actuator model and data for the active suspension system, as a single wheel station, has been taken from (Mehrishi 2007).

The individual actuator model has been used in a half vehicle model with three axles as shown in Figure 3. The wheel stations have been numbered with the front one being number 1 . The bump stops are shown and the tyres are modelled with by a linear spring $k_{t}$, which is capable of leaving the ground and the unsprung, axle, mass is given by $m_{u}$. The list of

\begin{tabular}{|c|c|}
\hline Symbol & Explanation \\
\hline$m_{s}$ & Vehicle sprung mass \\
\hline$m_{u_{i}}$ & Vehicle unsprung mass for $i$-th axle \\
\hline$q_{i}$ & Servo flow for $i$-th damper \\
\hline$z_{s}$ & Sprung mass vertical displacement \\
\hline$z_{u_{i}}$ & Axle vertical displacement for $i$-th axle \\
\hline$z_{t_{i}}$ & $\begin{array}{l}\text { Tyre verical displacement for } i \text {-th } \\
\text { wheel }\end{array}$ \\
\hline$z_{s_{i}}$ & Spring displacement for $i$-th wheel \\
\hline$z_{g_{i}}$ & Vertical ground displacement \\
\hline$v$ & Vehicle forward velocity \\
\hline$x_{p}$ & Preview distance \\
\hline$i$ & Wheel/axle/suspension index $\{1,2,3\}$ \\
\hline$k_{t}$ & Tyre stiffness \\
\hline$k_{d}$ & Spring stiffness \\
\hline$k_{b}$ & Bump-stop stiffness \\
\hline$R$ & Damper valve restriction \\
\hline$a_{p}$ & Lower piston area \\
\hline$a_{d}$ & Top piston area \\
\hline$z_{s_{i}}$ & $i$-th spring vertical displacement \\
\hline$Z_{\text {sus }}$ max & Suspension maximum displacement \\
\hline$x_{i}$ & Axle distance from C.G. \\
\hline$I_{s}$ & Sprung mass inertia \\
\hline$\Lambda\left(z_{g_{i}}\right)$ & Tyre force \\
\hline$P_{1}^{i}$ & $\begin{array}{l}\text { Pressure at top section for } i \text {-th suspen- } \\
\text { sion }\end{array}$ \\
\hline$P_{2}^{i}$ & $\begin{array}{l}\text { Pressure at lower section for } i \text {-th sus- } \\
\text { pension }\end{array}$ \\
\hline
\end{tabular}
symbols is shown in Table 1.

For the vehicle sprung mass the following equation applies (body bouncing motion):

$m_{s} \ddot{z}_{s}=-\left(F_{s_{1}} x_{1}+F_{s_{2}} x_{2}+F_{s_{3}} x_{3}\right)+m_{s} g$

For the vehicle sprung mass the following equation applies (body pitch motion):

$I_{S} \ddot{\theta}=F_{s_{1}} x_{1}+F_{S_{2}} x_{2}+F_{S_{3}} x_{3}$

For the vehicle unsprung mass the following equation applies:

$m_{u_{i}} \ddot{z}_{u_{i}}=F_{s_{i}}+\left(\frac{m_{u}}{i}\right) g-k_{t} \Lambda\left(z_{g_{i}}\right)$ 
The suspension motion displacements, velocities and accelerations are given from for each wheel station $i=\{1,2,3\}$ from the following equations:

$$
\begin{array}{r}
\left\{\begin{array}{l}
z_{s_{i}}=z_{s}-x_{i} \theta \\
z_{w_{i}}=z_{s_{i}}-z_{u_{i}}
\end{array}\right. \\
\left\{\begin{array}{l}
\dot{z}_{s_{i}}=\dot{z}_{s}-x_{i} \dot{\theta} \\
\dot{z}_{w_{i}}=\dot{z}_{s_{i}}-\dot{z}_{u_{i}}
\end{array}\right. \\
\left\{\begin{array}{l}
\ddot{z}_{s_{i}}=\ddot{z}_{s}-x_{i} \ddot{\theta} \\
\ddot{z}_{w_{i}}=\ddot{z}_{s_{i}}-\ddot{z}_{u_{i}}
\end{array}\right.
\end{array}
$$

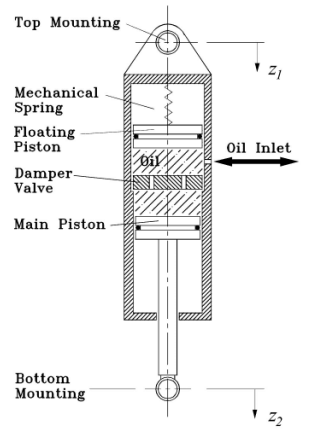

Figure 2. Active Suspension Actuator.

Based on the flow continuities the following expressions are valid for each actuator:

$$
\begin{aligned}
& q_{i} R=\dot{z}_{s_{i}} a_{d} R+\left(P_{1}^{i}-P_{2}^{i}\right) \\
& P_{1}^{i}=\frac{z_{s_{i}} k_{d}}{a_{d}}
\end{aligned}
$$

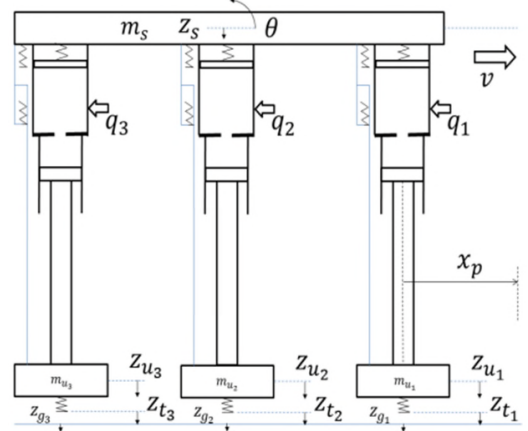

Figure 3. Axle Forces for a dual bump road surface.

\section{VEHICLE MODELLING SIMULATION}

Firstly, the vehicle is simulated for the same input profile with a passive suspension for all three axles. Thereafter the vehicle is run for the same road displacement (disturbance) with a proportional gain controller with the ride height set to zero for all three axles and lastly with the addition of a sprung mass acceleration feedback for all three axles.

The axle controllers are local to the axle and fixed to the three different settings for three vehicle simulation runs. The block diagram in Figure 7, represents the local controllers together with the feedforward Fuzzy Sugeno control architecture (with/without terrain preview).
The results for running the vehicle for the same road input for three different suspension conditions (i.e. passive suspension, and active suspension) are summarised in Figures, 4,5 and 6. Note that the road input $z_{g_{i}}<0$ for bumps and positive for portholes as indicated in Figure 4.
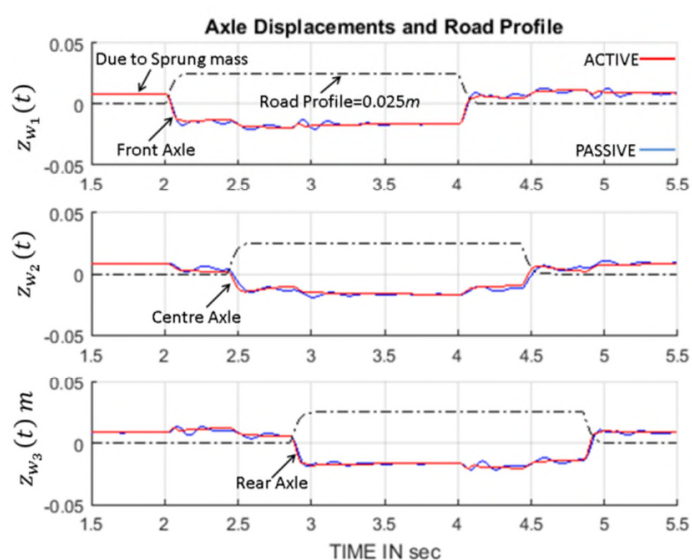

Figure 4. Without Preview: Axle Displacements and Road Profile for axles 1,2,3 respectively (Passive and Active Suspension).

The body sprung mass is much more effective when appropriately tuned with the ride height local controller $K_{p_{i}}$ and the sprung mass acceleration at point 1 gain $K_{q_{i}}$. For the results shown in Figures 5,6 and 7 the local controllers for each axle are identical.
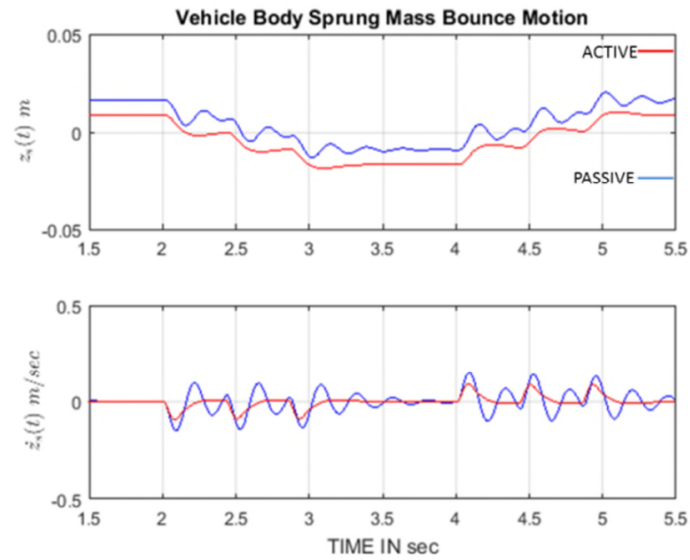

Figure 5. Without Preview: Vehicle Sprung Mass Characteristics (Passive and Active Suspension).
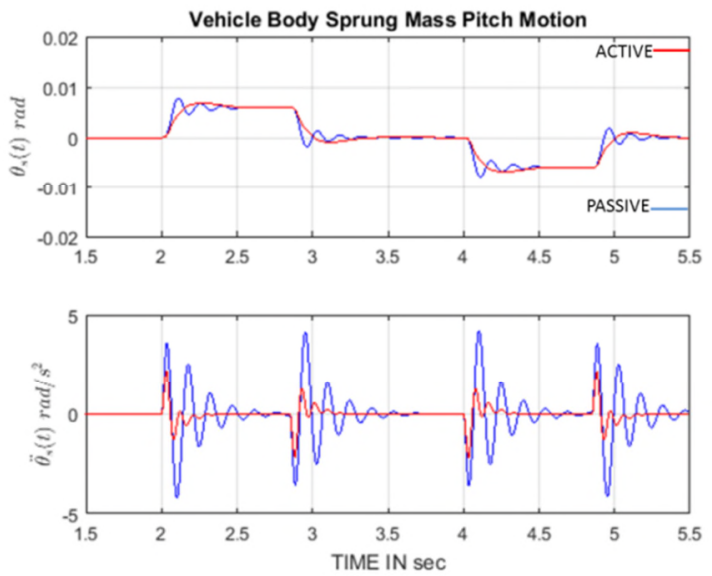

Figure 6. Without Preview: Vehicle Sprung Mass Pitch Motion (Passive and Active Suspension). 
Clearly the active suspension for all three axles results in a reduced pitch acceleration with an improved settling time as shown in Figure 6.

\section{SUGENO FUZZY LOGIC STRATEGY}

The Sugeno fuzzy inference design framework was utilised for two separate cases: Firstly, it was used to predict bumpstops using the look ahead sensor while running a vehicle model in advance of the real vehicle reaching the bumps. Secondly, it was used for the design of the feedforward fuzzy Sugeno controller with and without a preview sensor.

For both cases the local conventional control units are active. For the second case the fuzzy Sugeno feedfoward based control strategy wass implemented with the option of the preview being on or off for a range of parametric preview distance settings. In (Kiyotsugu 2003) the authors preduced a servo-motor preview controller for a servomechanism. The control method was based on an $H^{\infty}$ with preview for different preview times. For all inputs the system will settle within 60 steps while the preview was ranged between one to 10 steps.

The method in (Kiyotsugu 2003) indicated that as the preview was increased the system response was improved, however the input was a step and was lacking the complexity of the multi-axles responses affecting each other. To address this specific challenge the feedforward fuzzy Sugeno controllers used in this paper have a similarity with the control law shown in (Kiyotsugu 2003), whereby $(T)$ is the time corresponding to the preview time in relation to the running time $(t)$ :

$u_{i}^{f f}(t)=w_{i}^{1} \cdot F_{s}^{z}\left(z_{g_{i}}^{f}(T)\right)+w_{i}^{2} \cdot F_{s}^{\theta}\left(z_{g_{i}}^{f}(T)\right)$

$w_{i}^{1}, w_{i}^{2}$ : are the control weights for the fuzzy Sugeno vertical acceleration and body pitch acceleration controllers: $F_{s}^{z}\left(z_{g_{i}}^{f}(T)\right), F_{s}^{\theta}\left(z_{g_{i}}^{f}(T)\right)$ respectively. The local sensors per suspension unit (feedback, active suspension) is tuned without considering the preview data.

Hence the overall control law for each axle $(i)$ is given from:

$$
\begin{gathered}
\tilde{u_{l}}(t)=u_{f b}(t)+u_{i}^{f f}(t)= \\
\tilde{u_{l}}(t)=\ddot{z_{s_{l}}}(t) \cdot K_{a}+\left(z_{w_{i}}^{r e f}(t)-z_{w_{i}}(t)\right) \cdot K_{p}+ \\
\left\{w_{i}^{1} \cdot F_{S}^{z}\left(z_{g_{i}}^{f}(T)\right)+w_{i}^{2} \cdot F_{S}^{\theta}\left(z_{g_{i}}^{f}(T)\right)\right\}
\end{gathered}
$$

It is expected that as the vehicle speed increases the preview controller will offer a better vehicle rynamic response.

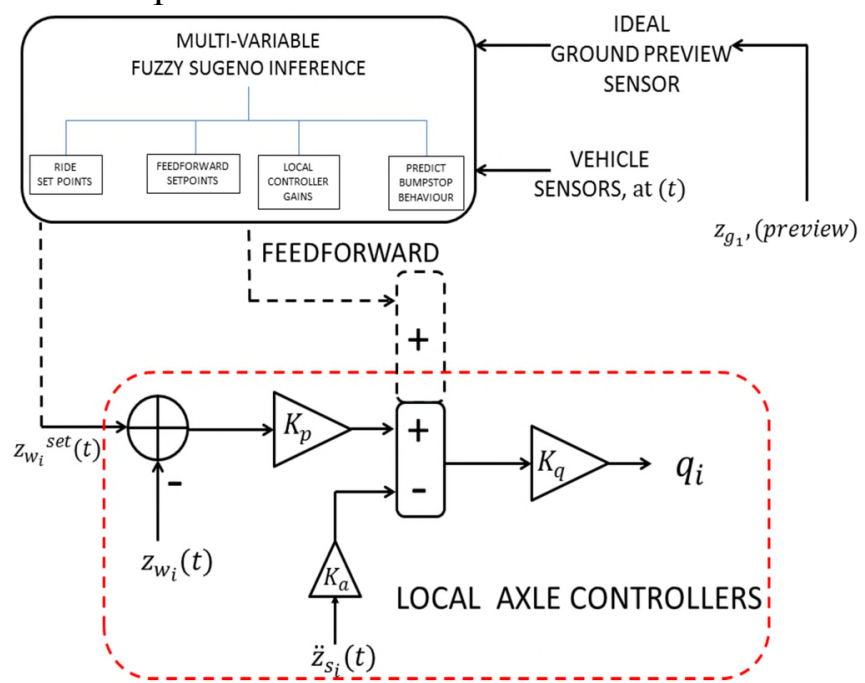

Figure 7. Fuzzy Sugeno Architecture with Local Controllers.

The Sugeno fuzzy inference was also the basis for predicting bumps by using the look-ahead sensor. This was mainly utilised by running a model of the vehicle ahead of time (in relation to the preview sensor). This provides useful information in relation to what would happen to the vehicle accelerations ahead of time. Figure 8 shows the membership functions and input/output characteristic of the bumbstop. Instead of simple logic the advantage for using the fuzzy logic method is smoother results as the suspension enters the bumpstop region.
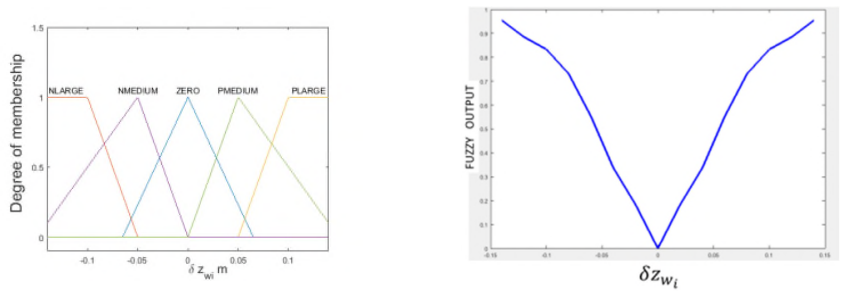

Figure 8. Fuzzy Sugeno Bumpstop Membership Functions and Output.
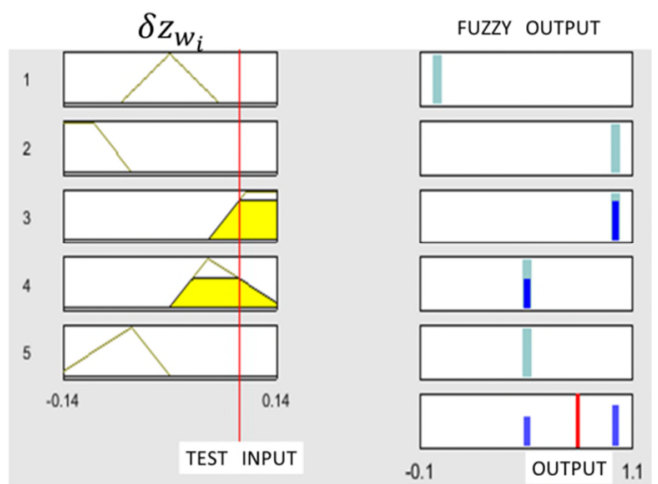

Figure 9. Fuzzy Sugeno Bumpstop Membership Functions and Singleton Outputs 
Regarding the fuzzy controllers: $F_{S}^{z}\left(z_{g_{i}}^{f}(T)\right)$ and $F_{s}^{\theta}\left(z_{g_{i}}^{f}(T)\right)$ model data were used to identify the number of necessary membership functions using the subtractive clustering method as also applied in (Economou et al. 2006). Therefore the fuzzy Sugeno based control system takes into account both the sprung mass acceleration in relation to the bumps as seen from the preview sensor. For a generic input variable vector: $z=\left[z_{r}^{i}, z_{m}^{i}, z_{f}^{i}\right]$, whereby $z_{r}^{i}$ represents the rear $i-t h$ sensor.

The Sugeno paremetrisation in the form of a rule base can be shown using the following expression (valid for $i=1$ ):

Rule i: IF $z_{r}^{1}(t)$ is $\mu_{i 1}\left(z_{r}^{1}\right) A N D z_{m}^{1}(t)$ is $\mu_{i 1}\left(z_{m}^{1}\right) A N D$.. .. $z_{r}^{1}$ is $\mu_{i 1}\left(z_{r}^{1}\right)$ THEN $y_{i}=b_{i}$

Therefore each Sugeno decision, (16), depends on both vertical sprung vehicle mass acceleration and sprung mass pitch angular acceleration. The membership functions: $\mu_{i 1}\left(z_{r}^{1}\right), \ldots, \mu_{i 1}\left(z_{m}^{1}\right)$ are optimised using the subtractive clustering method.

Lastly, in order to capture the fusion of multiple rules being fired at the same time (but potentially at a a different degree) we would need to utilize the defuzzification process described in equation (17):

$y^{*}(t)=\frac{\sum_{i=1}^{\max } \beta_{i} \cdot y_{i}(t)}{\sum_{i=1}^{\max } \beta_{i}}$

However, expression (17) can be rewritten in terms of the membership functions instead of the firing strengths, equation (18):

$y^{*}(t)=\frac{\sum_{i=1}^{\max }\left\{\mu_{i 1}\left(z_{r}^{1}\right) \wedge \mu_{i 2}\left(z_{m}^{1}\right) \wedge \mu_{i 3}\left(z_{r}^{1}\right)\right\} \cdot y_{i}(t)}{\sum_{i=1}^{\max }\left\{\mu_{i 1}\left(z_{r}^{1}\right) \wedge \mu_{i 2}\left(z_{m}^{1}\right) \wedge \mu_{i 3}\left(z_{r}^{1}\right)\right\}}$

The advantage of the expression in equation (18) is the instant response whereby combined with the feedforward and sensor look-ahead strategy offers a better overall response. Hence:

$y^{*}(t)=\left\{F_{s}^{z}\left(z_{g_{i}}^{f}(T)\right), F_{s}^{\theta}\left(z_{g_{i}}^{f}(T)\right)\right\}$

Figures 10(a),10(b), show the vertical and pitch sprung mass accelerations respectively in relation to the road filtered disturbance. A second order filter was used to smooth the disturbance profile and therefore remove and rapid transitions, equation 17 (where $\gamma$ is the dc correction gain). $\frac{z_{g_{i}}^{f}(s)}{z_{g_{i}}(s)}=\frac{\gamma \cdot \omega_{n}^{2}}{s^{2}+2 \zeta \omega_{n} \cdot s+\omega_{n}^{2}}$

The model, fuzzy estimated phase portrait is shown in Figure 10(c).

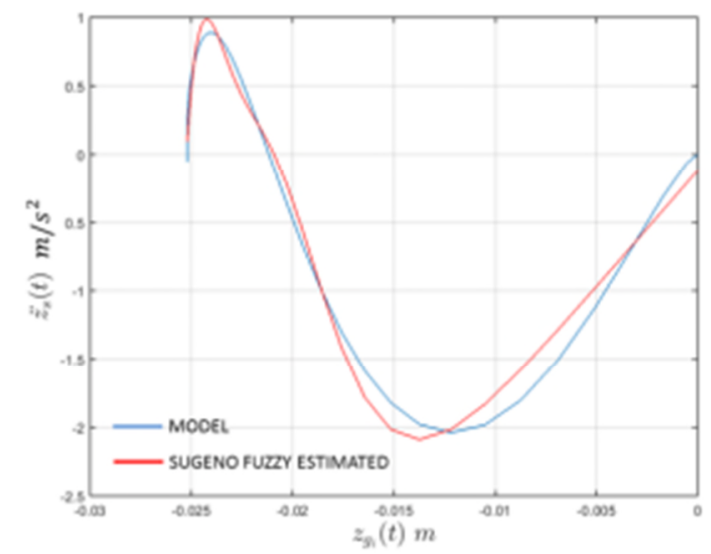

Figure 10 (a) Vehicle Sugeno Fuzzy Estimated and Reference Model Comparisons (Vertical Sprung Mass Acceleration and Road Disturbance).

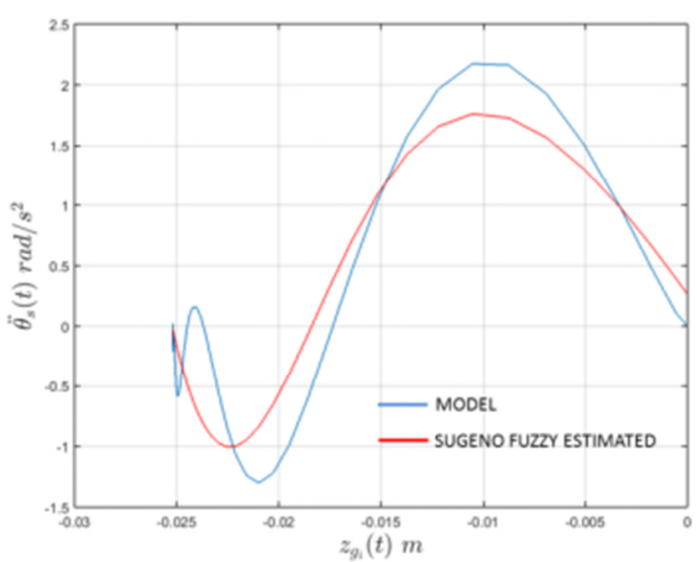

Figure 10 (b) Vehicle Sugeno Fuzzy Estimated and Reference Model Comparisons (Pitch Angular Sprung Mass Acceleration and Road Disturbance).

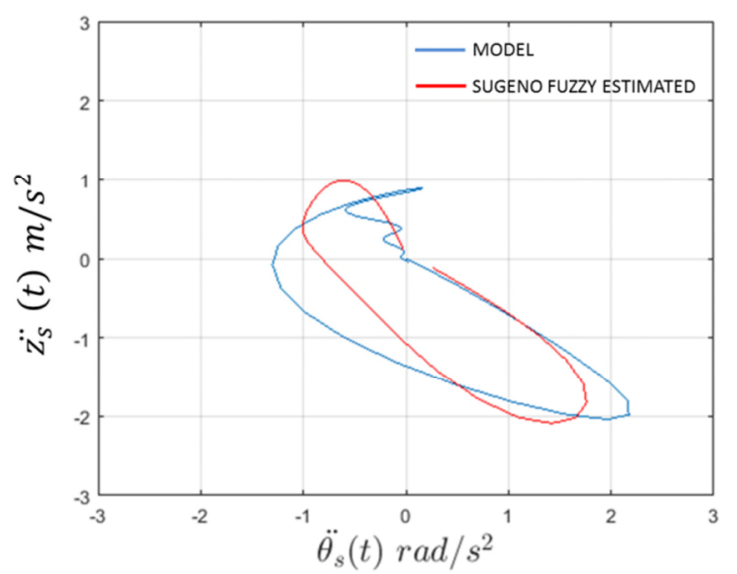

Figure 10(c) Phase Portrait - Vehicle Sugeno Fuzzy Estimated and Reference Model Comparisons (Vertical, Pitch Sprung Mass Accelerations).

\section{SIMULATION RESULTS}

The vehicle look-ahead sensor system prepares the tuned active suspension system in advance of the ground disturbances. Figure $\mathrm{xx}$ shows a range of 
simulation results. The blue line shows the reference/nominal accelerations as a benchmark. The remaining three (green, red, magenta), show the vehicle's response with the Sugeno fuzzy feedforward controllers for increasing preview distance. The Sugeno fuzzy controllers are locally optimised using the subtractive clustering algorithm (Economou et al. 2006).

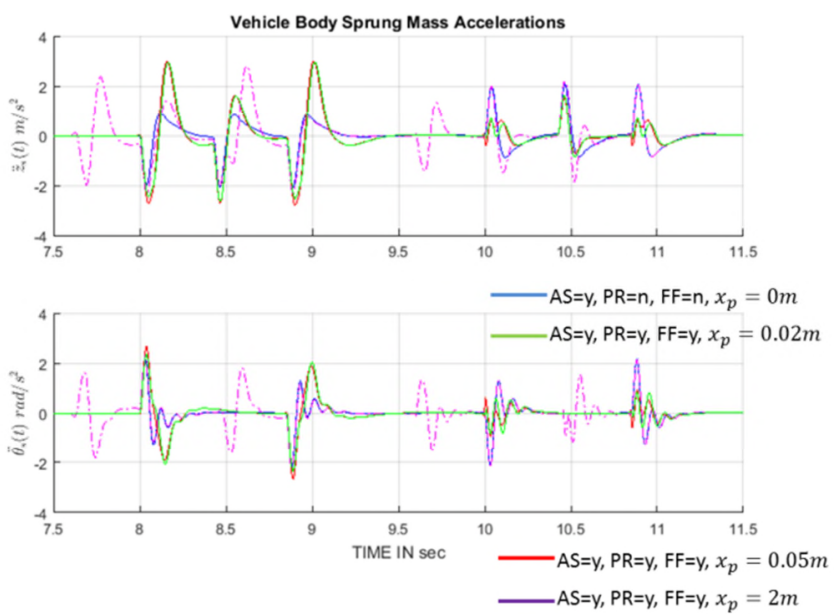

Figure 11. Vehicle Sprung Mass Accelerations with Variable Preview Distance $x_{p}$.

Clearly, the active suspension system performs well. The control strategy with the $2 \mathrm{~m}$ preview (fuzzy Sugeno feedforward) for the given distance overreacts thus creating controller based oscillations after $9.5 \mathrm{~s}$.

As per (Donohew et al. 2009) these need reducing in order to retain passenger comfort. The preview controller's response for a reduced preview distance has resulted in a much better range of accelerations especially for $x_{p}=0.02 \mathrm{~m}$.

\section{CONCLUSIONS}

A two-level intelligent based controller architecture is developed for a non-linear 3-axle single line vehicle model travelling on a rough terrain. The simulation results show the passive suspension, active suspension without and with a feedforward preview controller incorporating a prediction of bump stops ahead of the vehicle. The subtractive clustering optimisation algorithm was utilised to obtain the Sugeno fuzzy feedforward gains in relation to the road disturbances. The results showed that the look ahead distance is a critical parameter which affected the results.

For short look ahead distance the feedforward Sugeno fuzzy controllers could prepare the suspension and therefore the sprung mass vertical acceleration and pitch angular acceleration was improved.
However, as the model was run for much larger preview distances for the given vehicle velocity the preview feedforward Sugeno fuzzy controller was over-compensating thus resulting in adverse accelerations effects.

\section{REFERENCES}

Berrouila, L., 2008. Experimental and Theoretical Investigations of Active Suspension with Preview Control, MSc Thesis, Cranfield University, CDS Shrivenham.

Bo, F., 2010. Implementation of a fuzzy logic terrain preview controller on the active suspension rig, MSc Thesis, Cranfield University, SoE.

Donohew, B.E. \& Griffin, M.J. 2009. Motion Sickness with Fully Roll-Compensated Lateral Oscillation: Effect of Oscillation Frequency. Aviation, Space, and Environmental Medicine, 80 (2).

Economou, J.T. \& Colyer, R.E., 2006. Fuzzy-hybrid modelling of an Ackerman steered electric vehicle. ELSEVIER International Journal of Approximate Reasoning, 41 (3): 343-368.

Feng, G. 2006. A survey on analysis and design of Model-Based Fuzzy Control Systems. IEEE Transactions on Fuzzy Systems 14 (5): 676-697.

Kiyotsugu Takaba, 2003. A tutorial on preview control systems. SICE Annual Conference in Fukui, Fukui University, Japan.

Mehrishi, S., 2007. Active Suspension system with preview for off road military wheeled vehicles. MSc Thesis, Cranfield University, CDS Shrivenham.

Purdy, D. J. \& Bulman, D. N., 1997. An Experimental and Theoretical Investigation into the Design of an Active Suspension System for a Racing Car. I.Mech.E., Journal of Automobile Engineering (Part D), 211: 161-173.

Purdy, D, Mehrishi, S \& Berrouila, L., 2010. Design and Implementation of an active Suspension system with terrain Preview. Proc. of FISITA, F2010C126.

Purdy, D. J., Mehrishi, S. \& Berrouila, L., 2010. Design and implementation of an active suspension system with terrain preview, FISITA World Automotive Conference, Budapest.

Sharp, R. S., 1995. In J. P. Pauwellussen and H. B. Pacejka (eds), Preview control of active suspensions. In Smart Vehicles. Swets and Zeitlinger, 166-182.

Sher, E., Refael, S \& Luria, D., 1988. Agility and manoeuvrability as passive defence for armoured vehicles. Proc. Inst. of Mech. Eng. part D, Journal of Automobile Engineering, 202 (D1):65-70.

Takagi, T. \& Sugeno, M., 1985. Fuzzy identification of systems and its applications to modeling and control. IEEE transactions on systems, man, and cybernetics, (1): 116-132. 